\title{
QUALIDADE MICROBIOLÓGICA DO CONSUMO DE PESCADO CRU NA CULINÁRIA JAPONESA: SÍNTESE DE EVIDÊNCIAS
}

\author{
Microbiological quality of Raw Fish Consumption in Japanese Cuisine: Evidence Synthesis
}

\author{
Michely Calixto Olivetto ${ }^{1}$, Renato Ribeiro Nogueira Ferraz ${ }^{2}$
}

${ }^{1}$ Discente do curso de Gestão, Qualidade e Segurança em Alimentação para coletividade da Universidade Estácio de Sá - Campinas - SP. ${ }^{2}$ Docente do curso de Gestão, Qualidade e Segurança em Alimentação para coletividade da Universidade Estácio de Sá - Campinas - SP. E-mail: michely.olivetto@gmail.com

\section{RESUMO}

Introdução: Nas últimas décadas com a globalização, a alimentação tem sido motivo de preocupação em todos os países, ficando evidente os problemas relativos à sua qualidade para consumo e o impacto socioeconômico que pode vir a gerar. Apesar das várias qualidades do peixe, muito consumido na culinária japonesa, é considerado de alto risco por ser um alimento perecíveis e de fácil oxidação da gordura. Podendo assim, veicular microrganismos que fazem parte da microbiota natural do pescado, da água ou originária da má manipulação. Objetivo: Revisar a literatura buscando evidenciar os aspectos sobre a qualidade microbiológica encontrada no pescado cru dos alimentos da culinária japonesa (sushi e sashimi). Método: Trata-se de uma pesquisa exploratória baseada em uma revisão da literatura com síntese de evidências, por meio da ferramenta de procura Publish or Perish, em que foram selecionados os artigos de maior relevância. Conclusão: A literatura demonstra que o pescado na sua forma crua pode se tornar um veículo de microrganismos como: E. coli, Salmonella spp., S. aureus, vibrio spp. e principalmente a coliformes termotolerantes. Deste modo, é fundamental que os manipuladores tenham consciência da necessidade das boas práticas em todos os processos da cadeia de produção, principalmente quando o alimento não passa pela etapa de cocção, aonde ocorre a redução dessa carga microbiana, que pode multiplicar quando exposto a temperatura ambiente por muito tempo.

Palavras-chave: Segurança Alimentar. Restaurantes japoneses. Pescados crus. Manipulação de alimentos. Sushi. Sashimi.

\begin{abstract}
Introduction: In the last decades with globalization, food has been a cause of concern in all countries, with problems regarding the quality of consumption and the socioeconomic impact that it can generate. Despite the many qualities of the fish, much consumed in Japanese cuisine, it is considered high risk because it is a perishable food and easy oxidation of fat. In this way, it can transport microorganisms that are part of the natural microbiota of the fish, of the water or originated from the bad manipulation. Objective: To review the literature in order to highlight aspects of the microbiological quality found in raw fish from Japanese cuisine (Sushi and Sashimi). Method: It is an exploratory research based on a review of the literature with synthesis of evidence, through the search tool Publish or Perish, in which the articles of greater relevance were selected. Conclusion: The literature demonstrates that fish in their raw form can become a vehicle for microorganisms such as E. coli, Salmonella spp, S. aureus, Vibrio spp. and mainly to Thermotolerant Coliforms. In this way, it is fundamental that the manipulators are aware of the need of good practices in all processes of the production chain, especially when the food does not go through the cooking stage, where the reduction of this microbial load occurs, which can multiply when exposed to temperature for a long time.
\end{abstract}

Keywords: Food safety. Japanese restaurants. Raw fish. Food handling. Sushi. Sashimi.

\section{INTRODUÇÃO}


Nas últimas décadas com a globalização, a alimentação tem sido motivo de preocupação em todos os países, ficando evidente os problemas relativos à sua qualidade para consumo e o impacto socioeconômico que pode vir a gerar (BALBANI; BUTUGAN, 2001). A culinária japonesa tornou-se popular entre os brasileiros, tendo destaque para as preparações à base o pescado cru (sushi e sashimi), anteriormente restritas a restaurantes especializados em regiões com grande número de imigrantes asiáticos (GADELHA MALTA DE MOURA FILHO et al., 2007; VALLANDRO, 2010).

Dentre várias qualidades encontradas no peixe, estão a fácil digestibilidade, a presença de ácidos graxos poli-insaturados ômega 3, baixo teor de gordura e o alto teor proteico. No entanto, é considerado um alimento de alto risco por ser perecíveis e de fácil oxidação (SILVA; MATTÉ; MATTÉ, 2008). Podendo assim, veicular não só parasitas, como também outros patógenos, como bactérias que fazem parte da microbiota natural do pescado, da água ou originária da má manipulação (DE CASTRO PINHEIRO et al., 2006; MENEZES et al., 2006; MONTANARI et al., 2015).

A partir dos microrganismos encontrados no alimento, é possível ter uma noção da provável contaminação, que podem ser de origem fecal, potencial de deterioração do alimento ou de patógenos. Um dos principais indicadores são as bactérias do grupo coliformes totais e termotolerantes, utilizados na avaliação da qualidade sanitária do local de preparo (GADELHA MALTA DE MOURA FILHO et al., 2007; MARTINS, 2006).

Para a obtenção de um alimento de qualidade, é necessário tomar as devidas precauções com o tempo de armazenamento, a refrigeração, a manipulação e preparação. Resumindo, em toda a cadeia de processamento (da pesca a mesa do consumidor), tornando-se um risco a saúde se não manipulado da maneira correta (HUSS; REILLY; KARIM BEN EMBAREK, 2000; MONTANARI et al., 2015; SILVA; MATTÉ; MATTÉ, 2008). Um alimento seguro, em nível microbiológico, deve estar ausente de microrganismos em dose suficiente capaz de ocasionar uma intoxicação ou infecção alimentar. Segundo a OMS (Organização Mundial da Saúde), as doenças transmitidas por alimentos podem ser de natureza infecciosa ou tóxica, causadas pelo consumo de água ou alimento contaminado, podendo levar a sintomas leves como diarreia, vômitos e dores abdominais até condições graves de saúde (BAPTISTA; VENÂNCIO, 2003; SANTOS, R. M. DOS, 2006; VIEGAS, 2010). Com o intuito de fornecer informações atualizadas sobre o tema, que poderão ser utilizadas para a formulação de campanhas de esclarecimentos, buscou-se revisar a literatura sobre a qualidade microbiológica encontrada no pescado cru consumido na culinária japonesa.

\section{OBJETIVO}

Revisar a literatura buscando evidenciar os aspectos sobre a qualidade microbiológica encontrada no pescado cru dos alimentos da culinária japonesa (Sushi e Sashimi).

\section{MÉTODO}

Trata-se de uma pesquisa exploratória baseada em uma revisão da literatura com síntese de evidências, por meio da ferramenta de procura Publish or Perish, utilizando-se como descritores: segurança alimentar AND restaurantes japoneses AND pescados crus AND manipulação de alimentos AND sushi AND sashimi. Foram utilizados apenas artigos de maior relevância, levando em consideração o índice $\mathrm{h}$.

\section{RESULTADOS}

A revisão da literatura foi finalizada em 09 de março de 2018. Foram encontrados 15 artigos, dos quais apenas 7 foram revisados. Os demais artigos não atendiam o tema abordado.

\section{REVISÃO DA LITERATURA}

Santos (2006), analisou 20 amostras coletadas em 8 peixarias dos mercados municipais da cidade de São Paulo. Nas amostras foram pesquisados os microrganismos: Coliformes Termotolerantes, E. coli, Salmonella spp., S. aureus coagulase positiva e Vibrio spp., além da 
análise das estruturas dos locais de venda. Segundo os resultados da análise microbiológica, foram encontradas $20 \%$ das amostras com Coliformes Termotolerantes sendo apenas $10 \%$ com valores acima do preconizado pela legislação, $10 \%$ de E. coli com valores abaixo do preconizado pela legislação, $15 \% \operatorname{com} S$. aureus sendo apenas $5 \%$ acima do preconizado e $15 \%$ de Vibrio spp. Porém apenas $25 \%$ das amostras tinham valores acima do preconizado pela Anvisa.

Martins (2006), coletou 200 gramas de preparações a base de pescado cru de 20 estabelecimentos, sendo 8 especializados na culinária e 12 não especializados. Nas amostras foram pesquisados os microrganismos: Coliformes Termotolerantes, E. coli, S. aureus, Salmonella spp., vibrio spp., Bacillus cereus, Aeromonas spp. Segundo os resultados da análise microbiológica $50 \%$ das amostras coletadas apresentaram contagem de coliformes termotolerantes acima do limite permitido pela legislação brasileira, $45 \%$ de E. coli sendo $50 \%$ em estabelecimentos especializados, $45 \%$ apresentaram $S$. aureus sendo $15 \%$ com contagem acima do permitido, $35 \%$ de Vibrio, $75 \%$ das amostras apresentaram Aeromonas e não foi encontrado a presença de Salmonella spp. As amostras que se encontravam no balcão fora de refrigeração foram quantificadas com maior número de Coliformes Termotolerantes. Segundo o autor, uma análise mais rigorosa indicava que nenhuma das amostras era segura para o consumo, pois todas apresentavam pelo menos um fator de risco a saúde.

De Castro Pinheiro et al. (2006), realizaram 6 coletas em 5 estabelecimentos que comercializam comida japonesa em fortaleza, à procura de Salmonella e Coliformes Termotolerantes. $30 \%$ das amostras apresentaram valores acima do permitido para Coliformes Termotolerantes, 4 amostras com Salmonella e foram encontrados também E. coli, Enterobacter Aerogenes, Citrobacter Freundii e Klebsiella sp.

Silva et al. (2008), avaliaram 20 amostras em um período de 6 meses em 5 feiras na cidade de São Paulo. Dentre as 20 amostras analisadas 65\% continham microrganismos patógenos e ou potencialmente. Sendo $25 \%$ de Coliformes Termotolerantes com valores superiores ao preconizado, $10 \%$ de E. Coli, 15\% Vibrio, S. aureus foi isolado, porém dentro dos limites de tolerância. Não foi encontrada Salmonella presente nas amostras.

No estudo de Vallandro (2010), foram feitas inspeções sanitárias e coletas de amostras de sashimi em 6 restaurantes especializados em culinária japonesa. Nas amostras foram pesquisados os microrganismos: Coliformes, Staphylococcus coagulase, Vibrio e Salmonella. $\mathrm{Na}$ análise das conformidades dos restaurantes todos foram considerados adequados na avaliação global dos itens. $25 \%$ das amostras apresentaram níveis de Coliformes Termotolerantes acima do estabelecido e impróprios para consumo. Não foram encontrados Salmonella e Vibrio.

Santos et al. (2012), avaliaram 35 amostras em 7 restaurantes de grande movimento em Aracaju - SE. Foram analisadas amostras a procura de Salmonella, Staphylococcus e coliformes termotolerantes. Foram encontrados nas amostras $80 \%$ de Coliformes Termotolerantes e 11,4\% $S$. aureus acima do limite permitido pela legislação brasileira. Não foram encontradas nas amostras Salmonella.

Montanari et al. (2015), coletaram 15 amostras em 3 restaurantes especializados na culinária japonesa no município de Ji-Paraná Ro. Foram analisadas amostras a procura de Salmonella, Staphylococcus, Listeria Monocytogenes, Coliformes Termotolerantes e Microrganismos Mesófilos. Foram encontrados valores acima do permitido pela legislação vigente de Mesófilos em 26,7\%, Staphylococcus dentro dos limites permitidos e presença de Listeria monocytogenes em algumas amostras. Dos três locais de coleta, apenas um estava com os níveis de Coliformes Termotolerantes de todas as amostras dentro do recomendado.

\section{SÍNTESE DE EVIDÊNCIAS}

A literatura demonstra que o pescado na sua forma crua pode se tornar um veículo de microrganismos como: E. coli, Salmonella spp., S. aureus, Vibrio spp. e principalmente a Coliformes Termotolerantes. Deste modo, é fundamental que os manipuladores tenham 
consciência da necessidade das boas práticas em todos os processos da cadeia de produção, principalmente quando o alimento não passa pela etapa de cocção, aonde ocorre a redução dessa carga microbiana, que pode multiplicar quando exposto a temperatura ambiente por muito tempo.

\section{REFERÊNCIAS}

BALBANI, A. P. S.; BUTUGAN, O. Contaminação biológica de alimentos. Pediatria, v. 23, n. 4, p. 320-328, 2001.

BAPTISTA, P.; VENÂNCIO, A. Os perigos para a segurança alimentar no processamento de alimentos. [S.1.]: Forvisão: consultoria em formação integrada, lda., 2003. Disponível em: $<$ https://repositorium.sdum.uminho.pt/bitstream/1822/33398/1/document_2748_1.pdf $>$.

Acesso em: 5 out. 2017.

DE CASTRO PINHEIRO, H. M. et al. Salmonella sp. e coliformes termotolerantes em sushi e sashimi comercializados na cidade de Fortaleza-Ceará. Bol. Téc. Cient. CEPENE, v. 14, n. 1, p. 23-31, 2006.

GADELHA MALTA DE MOURA FILHO, L. et al. Enumeração e pesquisa de Vibrio spp. e coliformes totais e termotolerantes em sashimis de atum e vegetais comercializados na região metropolitana do Recife, Estado de Pernambuco. Acta Scientiarum. Technology, v. 29, n. 1, p. 85-90, 2007.

HUSS, H. H.; REILLY, A.; KARIM BEN EMBAREK, P. Prevention and control of hazards in seafood. Food Control, v. 11, n. 2, p. 149-156, 1 abr. 2000.

MARTINS, F. DE O. Avaliação da qualidade higiênico-sanitária de preparações (sushi e sashimi) a base de pescado cru servidos em bufês na Cidade de São Paulo. USP, São Paulo$S P, 2006.142$. 2006. PhD Thesis - Dissertação de mestrado)-Universidade de São Paulo, Faculdade de Saúde Pública, SP, 2006.

MENEZES, F. G. R. et al. Salmonella e staphylococcus coagulase positiva em sushis e sashimis comercializados na cidade de Fortaleza, Ceará. Anais do II Simpósio de Controle do Pescado-SIMCOPE [internet], 2006. Disponível em:

$<$ http://www.simcope.com.br/II_Simcope/pdf/402.pdf>. Acesso em: 28 set. 2017.

MONTANARI, A. S. et al. Avaliação da qualidade microbiológica de sashimis de salmão, preparados e comercializados em restaurantes japonês no município de Ji-Paraná-Ro. South American Journal of Basic Education, Technical and Technological, v. 2, n. 1, p. 4-16, 2015.

SANTOS, A. A. et al. Avaliação da qualidade microbiológica de sushi comercializado em restaurantes de Aracaju, Sergipe. Scientia Plena, v. 8, n. 3 (a), p. 1-5, 2012.

SANTOS, R. M. DOS. Avaliação da qualidade higiênico-sanitária de peixes comercializados em mercados municipais da cidade de São Paulo, SP. 2006. PhD Thesis - Universidade de São Paulo, 2006.

SILVA, M. L. DA; MATTÉ, G. R.; MATTÉ, M. H. Aspectos sanitários da comercialização de pescado em feiras livres da cidade de São Paulo, SP/Brasil. Revista do Instituto Adolfo Lutz (Impresso), v. 67, n. 3, p. 208-214, 2008.

VALLANDRO, M. J. Avaliação da qualidade microbiológica de sashimis a base de salmão, preparados em restaurantes especializados em culinária japonesa na cidade de Porto Alegre- 
RS. 2010. Disponível em: <http://www.lume.ufrgs.br/handle/10183/28854>. Acesso em: 28 set. 2017.

VIEGAS, S. J. Alterações do estado de saúde associadas à alimentação: contaminação microbiológica dos alimentos. Instituto Nacional de Saúde Doutor Ricardo Jorge, IP, 2010. Disponível em: <http://repositorio.insa.pt/handle/10400.18/143>. Acesso em: 3 out. 2017. 